\title{
FUSING THE COORDINATES OF QUANTUM SUPERSPACE
}

\author{
Peter BOUWKNEGT ${ }^{1}$, Jim McCARTHY ${ }^{1}$ and Peter van NIEUWENHUIZEN ${ }^{2}$ \\ ${ }^{1}$ Department of Physics and Mathematical Physics \& \\ Institute for Theoretical Physics \\ University of Adelaide \\ Adelaide, SA 5005, Australia \\ 2 Institute for Theoretical Physics \\ State University of New York \\ Stony Brook, NY 11794-3800, USA
}

\begin{abstract}
We introduce the notion of a fused quantum superplane by allowing for terms $\theta \theta \sim x$ in the defining relations. We develop the differential calculus for a large class of fused quantum superplanes related to particular solutions of the Yang-Baxter equation.
\end{abstract}

ADP-96-35/M46

ITP-SB-96-60

hep-th/9611067

November 1996 
In the conventional approach to quantum superplanes (see e.g. [1] for a review) one starts with a set of quadratic relations between a set of $M$ bosonic coordinates $x^{\mu}$ and $N$ fermionic coordinates ${ }^{1} \theta^{\alpha}$

$$
\begin{aligned}
\theta^{\alpha} \theta^{\beta} & =\widehat{R}_{\gamma \delta}^{\alpha \beta} \theta^{\gamma} \theta^{\delta}, \\
x^{\mu} \theta^{\alpha} & =\widehat{R}_{\beta \nu}^{\mu \alpha} \theta^{\beta} x^{\nu}, \\
x^{\mu} x^{\nu} & =\widehat{R}_{\rho \sigma}^{\mu \nu} x^{\rho} x^{\sigma} .
\end{aligned}
$$

On introducing coordinates $z^{a}=\left\{x^{\mu}, \theta^{\alpha}\right\}(\mu=1, \ldots, M, \alpha=1, \ldots, N)$ these may be written as

$$
z^{a} z^{b}=\widehat{R}_{c d}^{a b} z^{c} z^{d}
$$

The quantum superplane $\mathcal{A}_{q}$, determined by $\widehat{R}$, is then defined to be the quadratic algebra obtained by modding out the relations (2) from the free associative algebra generated by the $z^{a}$. One may consider $\mathcal{A}_{q}$ which are a flat deformation of the usual $(M \mid N)$-superspace: the matrix $\widehat{R}$ then depends on a set of deformation parameters, here collectively denoted by $q$, such that $q \rightarrow 1$ corresponds to the classical limit $\widehat{R}_{c d}^{a b}(q=1)=(-1)^{|a||b|} \delta_{d}^{a} \delta_{c}^{b}$; moreover, the flatness condition is the statement that $\mathcal{A}_{q}$ is isomorphic to $\mathcal{A} \otimes \mathbb{C}(q)$ as a $\mathbb{C}(q)$-module, where $\mathcal{A}=\mathcal{A}_{q=1}$ is the underformed superplane. In most examples $\widehat{R}$ is taken to be a solution of the Yang-Baxter equation (YBE).

Some time ago, before the advent of quantum groups, it was suggested that the coordinates $x^{\mu}$ and $\theta^{\alpha}$ of superspace are not on equal footing, but rather that the coordinates $x^{\mu}$ of spacetime are "composites" of the more fundamental fermionic variables $\theta^{\alpha}[2]$. To explore this idea a point particle model was constructed, with Lagrangian $\mathcal{L}=\bar{\theta}(\gamma \cdot x)^{-1} \dot{\theta}+\mathcal{L}_{1}(x, \dot{x}, \theta)$, where $\mathcal{L}_{1}$ contains terms quadratic in $\dot{x}$ such that the only second class constraints were those for the conjugate momenta of $\theta^{\alpha}$. The Dirac brackets for the fermionic coordinates ${ }^{2}$ were found to realize the compositeness of the $x^{\mu}$

$$
\left[\theta^{\alpha}, \theta^{\beta}\right]_{D}=\gamma_{\mu}^{\alpha \beta} x^{\mu}
$$

1 The distinction between bosonic and fermionic coordinates may seem artificial in the general context of [1], we make it since we will be imposing the corresponding $\mathbb{Z}_{2}$ grading for physical reasons.

2 The remaining brackets were $\left[x^{\mu}, x^{\nu}\right]_{D}=\left[x^{\mu}, \theta^{\alpha}\right]_{D}=0,\left[x^{\mu}, p_{\nu}\right]_{D}=\delta_{\nu}^{\mu},\left[\theta, p_{\mu}\right]_{D}=$ $\frac{1}{2} \gamma_{\mu}(\gamma \cdot x)^{-1} \theta$ and $\left[p_{\mu}, p_{\nu}\right]_{D}=\frac{1}{2} \bar{\theta} \gamma_{\mu \nu \rho} \theta \frac{x^{\rho}}{x^{4}}$. 
However, whereas Lorentz generators were constructed, yielding the conventional Lorentz algebra, the authors of [2] were unable to construct translation generators $P_{\mu}$ satisfying $\left[P_{\mu}, P_{\nu}\right]_{D}=0$ and acting on the $x^{\mu}$ as usual, $\left[P_{\mu}, x^{\nu}\right]_{D}=\delta_{\mu}^{\nu}$. The proposal was incomplete and very speculative, and no further work was done on the subject.

In this letter, we reconsider (3) in the context of quantum deformations of the superplane. Motivated by [2] we generalize (2) by allowing for a term linear in coordinates

$$
z^{a} z^{b}=\widehat{R}_{c d}^{a b} z^{c} z^{d}+T_{c}^{a b} z^{c}
$$

Such a term preserves the dimensions -1 of $x^{\mu}$ and $-\frac{1}{2}$ of $\theta^{\alpha}$ provided

$$
T_{c}^{a b}=0, \quad \text { unless } a=\alpha, b=\beta, c=\mu,
$$

which we will henceforth assume. An equation similar to (4) was considered in the context of braided Lie algebras $[3,4]$, the $z^{a}$ then corresponding to the generators of a deformed Lie algebra. However, in that context the $T_{c}^{a b}$ tend to the structure constants of the Lie algebra in the classical limit whereas in our approach we allow the limit to be zero. Further, the conditions that arise on $\widehat{R}$ and $T$ are different in the two contexts. The fused quantum superplane $\mathcal{A}_{q}$, determined by $\widehat{R}_{c d}^{a b}$ and $T_{c}^{a b}$, is then defined to be the quadratic algebra obtained by modding out the relations (4) from the free associative algebra generated by the $z^{a}$. Again we are interested in the case where $\mathcal{A}_{q}$ is a flat deformation of the usual quantum superplane. We find that the flatness conditions in the fused case are more restrictive than in the unfused case since by exploring the associativity conditions one is led to compatibility relations between the various components of $\widehat{R}$. In addition we impose the natural condition

$$
\widehat{R}_{c d}^{a a}=(-1)^{|a|} \delta_{c}^{a} \delta_{d}^{a}
$$

For definiteness we discuss a simple model first. Consider a $(2 \mid 2)$ superspace in $d=$ $(1,1)$ spacetime with lightcone coordinates $x^{+}, x^{=}, \theta^{+}$and $\theta^{-}$. Assuming the preservation of the Lorentz index structure (which is a natural assumption if we ultimately want to have a deformation of the Lorentz group acting on our fused quantum superplane) leads to an ansatz with eight free parameters. On demanding compatibility of the associativity relations such as $\left(\left(\theta^{+} \theta^{+}\right) \theta^{+}\right)=\left(\theta^{+}\left(\theta^{+} \theta^{+}\right)\right)$with the defining relations (4), it is clear that 
the complete set may be computed once the fundamental relations for two $\theta$ 's have been given. We find (cf. [5])

$$
\begin{array}{rlrl}
\theta^{+} \theta^{-} & =q \theta^{-} \theta^{+}, & \\
\theta^{+} \theta^{+}=\alpha x^{+}, & \theta^{-} \theta^{-}=\beta x^{=}, \\
x^{+} \theta^{+}=\theta^{+} x^{+}, & x^{=} \theta^{+}=q^{-2} \theta^{+} x^{=}, \\
x^{+} \theta^{-}=q^{2} \theta^{-} x^{+}, & x^{=} \theta^{-}=\theta^{-} x^{=}, \\
x^{+} x^{=}=q^{4} x^{=} x^{+} . &
\end{array}
$$

For $\alpha \neq 0$ or $\beta \neq 0$, one can scale $x^{+}$or $x^{=}$, respectively, to put the equations in a form with $\alpha=1$ or $\beta=1$. From this we see that the equations only contain one true deformation parameter, $q$. We will keep the $\alpha, \beta$ explicit, however, in order to discuss the ordinary superplane as the limit $\alpha, \beta \rightarrow 0$. Note that the equations (7) may be written in the form (4)-(6), where we may fix $T_{+}^{++}=2 \alpha$ and $T_{=}^{--}=2 \beta$. A generalization of the solution $(7)$ to $(N \mid N)$ superspace is easily constructed along the same lines. We will return to this generalization later.

We now return to a more general discussion of the fused quantum superplanes. First, we may also write (4) as

$$
\left(\delta_{c}^{a} \delta_{d}^{b}-\widehat{R}_{c d}^{a b}\right) z^{c} z^{d}=T_{c}^{a b} z^{c}
$$

In this form it is obvious that the relations (4) are the same as those corresponding to $\left(\widehat{R}^{\prime}, T^{\prime}\right)$ given by

$$
\begin{aligned}
\left(\delta_{c}^{a} \delta_{d}^{b}-\widehat{R}_{c d}^{a b}\right) & =\alpha^{a b}\left(\delta_{c}^{a} \delta_{d}^{b}-\widehat{R}_{c d}^{a b}\right) \\
T_{c}^{\prime a b} & =\alpha^{a b} T_{c}^{a b}
\end{aligned}
$$

for any choice of $\alpha^{a b} \neq 0$. A more general equivalence of this form is obtained by replacing $\alpha^{a b}$ by an invertible matrix $\alpha_{a^{\prime} b^{\prime}}^{a b}$. There are further equivalences corresponding to linear redefinitions of the coordinates. Since it is not our aim in this paper to give the most general quantum superplane up to these equivalences we will not explore this any further.

For invertible $\widehat{R}$-matrices, the relation (4) can also be written as

$$
z^{a} z^{b}=\left(\widehat{R}^{-1}\right)_{c d}^{a b} z^{c} z^{d}-\left(\widehat{R}^{-1}\right)_{c d}^{a b} T_{e}^{c d} z^{e}
$$

Although more general fused quantum superplanes are feasible, it is natural to impose that 
the equations (10) and (4) are related by

$$
\begin{aligned}
\left(\widehat{R}^{-1}\right)_{c d}^{a b} & =\lambda \widehat{R}_{c d}^{a b}+(1-\lambda) \delta_{c}^{a} \delta_{d}^{b}, \\
\left(\widehat{R}^{-1}\right)_{c d}^{a b} T_{e}^{c d} & =-\lambda T_{e}^{a b}
\end{aligned}
$$

for some $\lambda \neq 0$. Equivalently,

$$
\begin{aligned}
\lambda \widehat{R}^{2}+(1-\lambda) \widehat{R}-1 & =(\lambda \widehat{R}+1)(\widehat{R}-1)=0, \\
\lambda \widehat{R} T & =-T,
\end{aligned}
$$

i.e. $\widehat{R}$ satisfies a quadratic characteristic equation and $T_{c}^{a b}$ is an eigenvector of $\widehat{R}$ with eigenvalue $-1 / \lambda$ for all indices $c$. Moreover, any $(\widehat{R}, T)$-system satisfying (12) is equivalent under $(9)$ to an $(\widehat{R}, T)$-system satisfying

$$
\begin{gathered}
\widehat{R}^{2}=1, \\
\widehat{R} T=-T .
\end{gathered}
$$

Henceforth we will work with the 'gauge' (13), (14). Together with (6) we will refer to these conditions as the naturalness conditions on $(\widehat{R}, T)$. With the above naturalness conditions our differential calculus takes a particularly simple form, but the corresponding formulas for the more general gauge (12) can be easily worked out. ${ }^{3}$ Again we stress that there are additional relations on $\widehat{R}$ and $T$ coming from the compatibility of associativity of the coordinate ring with the defining relations (4). We do not know how to write these relations in closed formulas.

Both to construct interesting examples and to construct a differential calculus on fused quantum superplanes along the lines of $[6,7,8,9]$ it proves convenient to introduce an additional bosonic coordinate $z^{0}$ - commuting with all the other coordinates $z^{a}$ and which can be consistently specialized to a constant (as will be done at a later stage) - and rewrite (4) in terms of $z^{A} \equiv\left\{z^{0}, z^{a}\right\}=\left\{z^{0}, x^{\mu}, \theta^{\alpha}\right\}$ (cf. [3,4])

$$
z^{A} z^{B}=\widehat{\mathcal{R}}_{C D}^{A B} z^{C} z^{D}
$$

with

$$
\widehat{\mathcal{R}}_{C D}^{A B}=\left(\begin{array}{cccc}
1 & 0 & 0 & 0 \\
0 & 0 & \delta_{c}^{b} & 0 \\
0 & \delta_{d}^{a} & 0 & 0 \\
0 & \frac{1}{2} T_{d}^{a b} & \frac{1}{2} T_{c}^{a b} & \widehat{R}_{c d}^{a b}
\end{array}\right)
$$

3 The formulas in [6], for the bosonic 2-plane, correspond to the gauge $\lambda=q^{2}$. 
Here the matrix rows correspond to $(00),(0 b),(a 0)$ and $(a b)$, the columns similarly to (00), $(0 d),(c 0)$ and $(c d)$, respectively. The quantum superplane $\mathcal{A}_{q}$ extended by $z^{0}$ will be denoted by $\widehat{\mathcal{A}}_{q}$. The equations $(13)$ and $(14)$ for $(\widehat{R}, T)$ imply that $\widehat{\mathcal{R}}$ satisfies the same characteristic equation as $\widehat{R}$, i.e.

$$
\widehat{\mathcal{R}}^{2}=1
$$

A particularly interesting class of fused quantum superplanes is those for which $\widehat{\mathcal{R}}$ satisfies the Yang-Baxter equation

$$
(\widehat{\mathcal{R}} \otimes 1)(1 \otimes \widehat{\mathcal{R}})(\widehat{\mathcal{R}} \otimes 1)=(1 \otimes \widehat{\mathcal{R}})(\widehat{\mathcal{R}} \otimes 1)(1 \otimes \widehat{\mathcal{R}})
$$

or, in components,

$$
\widehat{\mathcal{R}}_{P Q}^{A B} \widehat{\mathcal{R}}_{K L}^{P R} \widehat{\mathcal{R}}_{R M}^{Q C}=\widehat{\mathcal{R}}_{P Q}^{B C} \widehat{\mathcal{R}}_{K R}^{A P} \widehat{\mathcal{R}}_{L M}^{R Q}
$$

Equation (18), together with (17), implies that $\widehat{\mathcal{R}}$ constitutes a representation of the permutation group. In terms of $\widehat{R}_{c d}^{a b}$ and $T_{c}^{a b}$, equation (19) reads

$$
\begin{aligned}
\widehat{R}_{p q}^{a b} \widehat{R}_{r m}^{q c} \widehat{R}_{k l}^{p r} & =\widehat{R}_{p q}^{b c} \widehat{R}_{k r}^{a p} \widehat{R}_{l m}^{r q} \\
\widehat{R}_{l q}^{a b} T_{m}^{q c}+\widehat{R}_{p q}^{a b} \widehat{R}_{r m}^{q c} T_{l}^{p r} & =\widehat{R}_{l m}^{a q} T_{q}^{b c}+\widehat{R}_{p q}^{b c} \widehat{R}_{l m}^{r q} T_{r}^{a p} \\
T_{p}^{a b} T_{l}^{p c}+\widehat{R}_{p q}^{a b} T_{l}^{p r} T_{r}^{q c} & =T_{q}^{b c} T_{l}^{a q}+\widehat{R}_{p q}^{b c} T_{r}^{a p} T_{l}^{r q} \\
T_{p}^{a b} T_{l}^{p c} & =2 T_{p}^{b c} T_{l}^{a p}+\widehat{R}_{p q}^{b c} T_{r}^{a p} T_{l}^{r q}
\end{aligned}
$$

The last two of these equations are trivially satisfied because of the preservation of dimension condition (5). Also note that the YBE is not preserved under the equivalence (9).

It is straightforward to check that the model in (7) (written in the form of equations (4)-(6)) satisfies these YBEs. In fact, suppose one takes (4) in its most general form for the $(2 \mid 2)$ plane which preserves grading, dimension, and Lorentz index structure (as discussed previously). Then imposing the YBE leaves (7) as the most general solution if one excludes those solutions (nonflat deformations) which impose spurious vanishing of products of the generators. One can push this slightly further. If we replace Lorentz index preservation by the naturalness conditions, and still only allow a deformation with $T_{\#}^{++}$and $T_{=}^{--}$nonzero, then the YBE still leads to the same unique solution. It is not clear how to make this analysis in a more general case, but this does show a rather pleasing consistency of the idea of naturalness at least for this case. 
Let us consider the case of the $(M \mid N)$ superplane; i.e. a plane with coordinates $\left(x^{\mu}, \theta^{\alpha}\right), \mu=1, \ldots, M, \alpha=1, \ldots, N$. We can look for solutions where the braiding matrix $\widehat{R}$ just has the effect of exchanging the coordinates up to some multiplicative factor, i.e.

$$
\widehat{R}_{c d}^{a b}=q_{a b} \delta_{d}^{a} \delta_{c}^{b}
$$

for some set of parameters $q_{a b}$. The condition (13) leads to

$$
q_{a b}=q_{b a}^{-1},
$$

thus, in particular, with $(6), q_{a a}=(-1)^{|a|}$, while (14) leads to

$$
q_{a b} T_{c}^{b a}=-T_{c}^{a b}
$$

The first equation in (20) is now automatically satisfied, while the second equation leads to the condition

$$
q_{a d}=q_{a b} q_{a c}, \quad \text { whenever } T_{d}^{b c} \neq 0 .
$$

One obvious solution to $(22)-(24)$ is the trivial one, i.e.

$$
q_{a b}=(-1)^{|a||b|}
$$

in which case the only constraint on $T_{\mu}^{\alpha \beta}$ (arising from $(23)$ ) is $T_{\mu}^{\alpha \beta}=T_{\mu}^{\beta \alpha}$. This corresponds to the situation studied in [2], i.e.

$$
\begin{aligned}
\theta^{\alpha} \theta^{\beta} & =-\theta^{\beta} \theta^{\alpha}+2 T_{\mu}^{\alpha \beta} x^{\mu}, \\
x^{\mu} \theta^{\alpha} & =\theta^{\alpha} x^{\mu} \\
x^{\mu} x^{\nu} & =x^{\nu} x^{\mu} .
\end{aligned}
$$

A less nontrivial solution, generalizing (7), is to consider an equal number of fermionic and bosonic coordinates $\left(x^{i}, \theta^{i}\right), i=1, \ldots, N$ and making the assumption that the relations (4) preserve the dimension in each direction $i$, i.e. $T_{x^{k}}^{\theta^{i} \theta^{j}}=\alpha_{i} \delta_{k}^{i} \delta_{k}^{j},\left(\alpha_{i} \neq 0\right)$, while all other $T_{c}^{a b}$ vanish. We immediately arrive at the following solution of (22)-(24)

$$
\begin{gathered}
\theta^{i} \theta^{j}=-q_{i j} \theta^{j} \theta^{i}+2 \alpha_{i} \delta^{i j} x^{i}, \\
x^{i} \theta^{j}=q_{i j}^{2} \theta^{j} x^{i} \\
x^{i} x^{j}=q_{i j}^{4} x^{j} x^{i} \\
-6-
\end{gathered}
$$


where $q_{i i}=1, q_{j i}=q_{i j}^{-1}$ and the $q_{i j}, i<j$ and $\alpha_{i}$ are arbitrary deformation parameters. We will refer to this solution as the fused quantum superplane $\mathcal{A}_{(N \mid N)}$. The quantum superplanes $\mathcal{A}_{(M \mid N)}$ for $M \neq N$ are defined by setting the appropriate coordinates (and deformation parameters) to zero in $\mathcal{A}_{(\max (M, N) \mid \max (M, N))}$. It is easily seen that the solutions (26) and (27), obtained by solving the Yang-Baxter equations, are fully compatible with the associativity constraints; i.e. imposing associativity does not lead to additional relations on the coordinates. In general we expect this to be the case for all solutions to (20) satisfying the naturalness conditions (6), (13) and (14).

Now we briefly discuss the differential calculus on a fused quantum superplane. We start with the formulation in terms of $\widehat{\mathcal{R}}$. One can introduce an exterior derivative $d$, differentials $d z^{A}$ and derivatives $\partial_{A}$, such that

$$
d^{2}=0, \quad d=d z^{A} \partial_{A}
$$

and for forms $\omega_{1} \in \bigwedge^{k} \widehat{\mathcal{A}}_{q}, \omega_{2} \in \bigwedge^{l} \widehat{\mathcal{A}}_{q}$

$$
d\left(\omega_{1} \omega_{2}\right)=\left(d \omega_{1}\right) \omega_{2}+(-1)^{k} \omega_{1}\left(d \omega_{2}\right)
$$

(We have chosen the convention where $d$ commutes with both the bosonic and fermionic coordinates of the quantum superplane.) Following the steps in [6] one finds that the following exchange formulas provide a consistent differential calculus on the fused quantum superplane $\widehat{\mathcal{A}}_{q}$

$$
\begin{aligned}
d z^{A} z^{B} & =\widehat{\mathcal{R}}_{C D}^{A B} z^{C} d z^{D} \\
d z^{A} d z^{B} & =-\widehat{\mathcal{R}}_{C D}^{A B} d z^{C} d z^{D} \\
\partial_{C} z^{A} & =\delta_{C}^{A}+\widehat{\mathcal{R}}_{C D}^{A B} z^{D} \partial_{B} \\
\partial_{C} \partial_{D} & =\widehat{\mathcal{R}}_{C D}^{B A} \partial_{A} \partial_{B} \\
d z^{A} \partial_{C} & =\widehat{\mathcal{R}}_{C D}^{A B} \partial_{B} d z^{D}
\end{aligned}
$$

To recover the differential calculus on $\mathcal{A}_{q}$, we have to specialize the formulas in (30) to the hyperplane $z^{0}=1$. Since $\widehat{\mathcal{R}}_{C D}^{0 B}=\delta_{C}^{B} \delta_{D}^{0}$ and $\widehat{\mathcal{R}}_{C D}^{A 0}=\delta_{D}^{A} \delta_{C}^{0}$, it is easily seen that the exchange formulas are consistent with the assignment $z^{0}=1, d z^{0}=0$. Also, when considering forms on the hyperplane $\mathcal{A}_{q}$, there is no need to define the derivative $\partial_{0}$. However, for consistency, we need to show that $\partial_{0}$ does not occur on the right hand side if it does not occur on the left hand side of (30). Again, this follows from the equations 
$\widehat{\mathcal{R}}_{C D}^{0 B}=\delta_{C}^{B} \delta_{D}^{0}$ and $\widehat{\mathcal{R}}_{C D}^{A 0}=\delta_{D}^{A} \delta_{C}^{0}$. (Sometimes, it can happen that $\partial_{0}$ can be eliminated by combining various equations of (30) in which $\partial_{0}$ does occur. In these cases, however, at least in examples, no independent equations on the other derivatives are obtained.) Thus, we conclude that (30) leads to a consistent differential calculus on $\mathcal{A}_{q}$.

A possible application of the foregoing might be a new kind of superspace supersymmetry and supergravity, or two-dimensional superconformal field theory, where the Grassmann thetas are replaced by our kind of Clifford thetas. One should then work out how the deformed supersymmetry algebra acts on the supercoordinates, using methods which are quite common in quantum groups. These ideas remain to be explored.

\section{Acknowledgements}

PvN would like to thank the Institute for Theoretical Physics of the University of Adelaide for hospitality while part of this work was being done. We would like to thank Jan de Boer for discussions. PB and JM acknowledge the support of the Australian Research Council, while PvN is supported by NSF grant PHY9309888.

\section{References}

[1] Yu. I. Manin, Quantum Groups and Non-Commutative Geometry, Publications CRM, Montréal, 1989; Comm. Math. Phys. 123 (1989) 163.

[2] J.H. Schwarz and P. van Nieuwenhuizen, Lett. Nuovo Cimento 34 (1982) 21.

[3] D.I. Gurevich, Sov. Math. Dokl. 33 (1986) 758.

[4] D. Bernard, Prog. Theor. Phys. Suppl. 102 (1990) 49; Phys. Lett. 260B (1991) 389.

[5] W.B. Schmidte, S.P. Vokos and B. Zumino, Zeit. für Physik C8 (1990) 249.

[6] J. Wess and B. Zumino, Nucl. Phys. (Proc. Suppl.) 18B (1990) 302.

[7] B. Zumino, Mod. Phys. Lett. A6 (1991) 1225.

[8] B. Zumino, in Proc. of the XIX International Conference on Group Theoretic Methods in Physics, Salamanca, Spain, 1992, hep-th/9212093).

[9] C. Chryssomalakos, P. Schupp and B. Zumino, Alg. Anal. 6 (1994) 252, (hep$\operatorname{th} / 9401141)$. 